\title{
Integration of Sensors and RFID's on Ultra-low-cost Paper-based Substrates for Wireless Sensor Networks Applications
}

\author{
Antonio Ferrer-Vidal, Amin Rida, Serkan Basat, Li Yang, and Manos M. Tentzeris \\ Georgia Electronic Design Center, School of Electrical and Computer Engineering, \\ Georgia Institute of Technology, Atlanta, GA 30332-0250, USA
}

E-mail:ferrer@ece.gatech.edu

\begin{abstract}
In this paper, an overview of novel integration approaches for improved performance UHF radio frequency identification (RFID) tags and embedded sensors and batteries is presented. Organic substrates, such as paper, that have been very rarely used in UHF and RF applications in the past and could potentially utilize inkjet printing techniques, are also thoroughly investigated for the realization of ultralow-cost RFID/Sensor tags for frequencies ranging from $13.56 \mathrm{MHz}$ up to $950 \mathrm{MHz}$ for the first time ever. The proposed technology could potentially revolutionize wearable and conformal wireless sensor networks (WSN).
\end{abstract}

Index Terms - RFID, Antennas, organic sensors, printed electronics, inkjet printing, thin film batteries, paper, wave polarization, integration, UHF.

\section{INTRODUCTION}

The demand for inexpensive, low power consumption and durable wireless nodes with sensing capabilities is driven by applications such as: item-level tracking of temperature sensitive products, pharmaceutical logistics, transport and storage of medical products (blood, medicines, vaccines...) or biosensing applications (biohazard materials, security...) among others. RFID is a compact wireless technology which does not require Line of Sight $(\operatorname{LoS})$ to communicate with the reader and allows simultaneously read/write from multiple tags, as well as an easy remote and selective activation of sensor devices based on their unique ID's. When combined with a power source it is possible to obtain significant benefits compared with passive tags or conventional bar codes in terms of read range. Moreover, a tag using the UHF RFID frequency band benefits from higher data rates than tags using the HF RFID band. Furthermore, its potential ability to be easily printed on organic substrates makes them an inexpensive candidate to significantly enhance the nodes in several Wireless Sensor Networks (WSN) applications.

In this paper, we briefly outline a novel approach to integrate RFID electronic components, namely antenna, power source, and IC with sensors in organic substrates, including paper, for frequencies up to $950 \mathrm{MHz}$ for the first time. Examples of current developments and undergoing research are also included in the paper.

\section{RFID ELECTRONIC COMPONENTS}

\section{A. SENSOR}

Recent developments in humidity sensors, based on ultrathin poly(anilinesulfonic acid) (SPANI) film [1], and pressure sensors fabricated on Liquid Crystal Polymer (LCP) [2] unfold a new field of opportunities in the organic sensor field. Additionally, development of organic thinfilm transistor (OTFT) sensors [3] opens up new possibilities in compact sensing systems, especially in biosensing applications. Such scenario leaves space for a conception of a mass production and integration process which will eventually reduce cost and will enable a largescale implementation of WSN's.

\section{B. ANTENNA}

\section{i. Bandwidth}

The UHF RFID band ranges from $860 \mathrm{MHz}$ to $930 \mathrm{MHz}$, typically $868 \mathrm{MHz}$ in Europe and 915 $\mathrm{MHz}$ in North America. This frequency band allows a high data rate over other bands typically used (for example the $13.56 \mathrm{MHz}$ ) and a high read range $(\sim 30$ feet for passive tags and $\sim 100$ feet for semi passive or active tags). A half wavelength antenna is typically used in RFID applications due to its omnidirectionality enabling the tags' communication with the RFID reader in any orientation and for a variety of environments. The fundamental topology of an integrated UHF RFID is demonstrated in Fig. 1. 


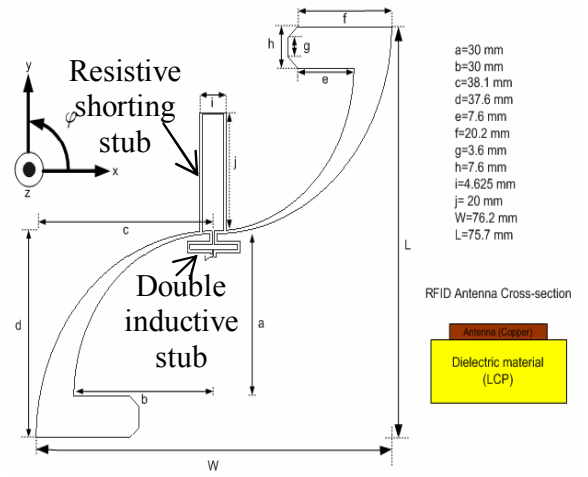

Fig. 1. RFID architecture

The dimensions of this antenna (3" x 3") allow for an integrated battery and/or sensor on the top left and bottom right corners of the antenna structure. The matching network (double inductive feed and shorting stub) controls the antenna's impedance and allows for an optimum power transfer from IC to antenna [4]. It has to be noted that the antenna has to be compact with efficiency close to $100 \%$

\section{ii. Harsh Environments}

Antennas in UHF RFID tags are linearly (vertical or horizontal) polarized. In the presence of metals and/or liquids (harsh environments) the transmitted/received electromagnetic waves undergo polarization changes. This causes a blockage of the communication with the reader. However; by using a dual antenna configuration with two identical in dimensions and shape bodies, such as the one shown in Fig. 2, one can greatly minimize this effect and also account for any misalignment of the tag that might cause a null in its radiation pattern that could potentially deteriorate the communication quality. This is especially critical in extreme "rugged" industrial and/or urban environments.
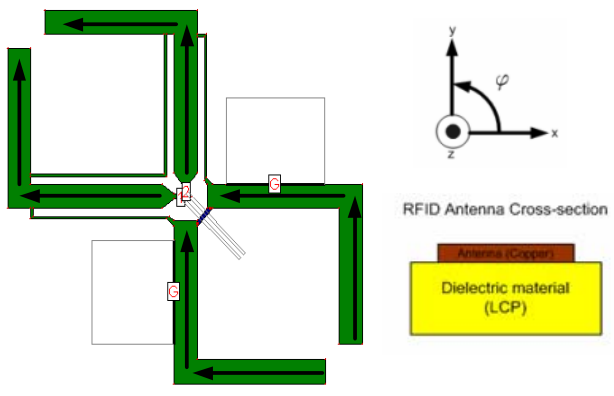

Fig. 2. Dual polarization antenna (arrows indicate current flow directions)

\section{POWER SOURCES}

One of the major concerns of WSN's is the limited lifetime of the batteries. The cost of replacing batteries in the nodes can be relatively high especially in remotely located nodes. The same issue is of major importance in active and semi-passive RFID tags. Three different power source types are available at the moment: power reservoirs, power distribution methods and power scavenging sources.

Among the power reservoir technologies investigated over the last years [5], rechargeable lithium thin film batteries seem to be the most suitable solution to be embedded in organic substrates due to their small thickness (less than 100 um). Such batteries have rechargeable capabilities which overcome short lifetime limitations, thus making them extremely useful for the drive of the sensors in active and semipassive RFID tags. Whereas the active tags solely utilize the battery to power the sensor and the IC, the semi-passive need a power distribution method (i.e. electromagnetic power transmission) to operate the IC, thus allowing the sensor to use the battery as an independent power source.

The main advantage of the reservoir source is that it eliminates the need for the label to collect energy from the reader, permitting the transmission of relatively large amounts of data over long distances ( $>100 \mathrm{ft}$.) while improving its signal-to-noise ratio. On the other hand, electromagnetic power transmission used with a battery allows for reduced battery consumption and an increased node's lifetime.

Among the power scavenging sources, thin film solar arrays play a major advantage in outdoor applications with difficult access and impossibility of using wires (i.e. industrial chemical WSN's)

\section{MODULE INTEGRATION}

In order to meet the ultra low cost demand for passive, semi-passive, and active RFID tags, a simple manufacturing process, such as conductive ink printing technology on organic substrates, is suggested. The ultimate goal is to have an all printed RFID tag (antenna, IC, battery, and sensor) on a low-cost environmental friendly paper. A block diagram of the tag is shown in (Fig. 3) 


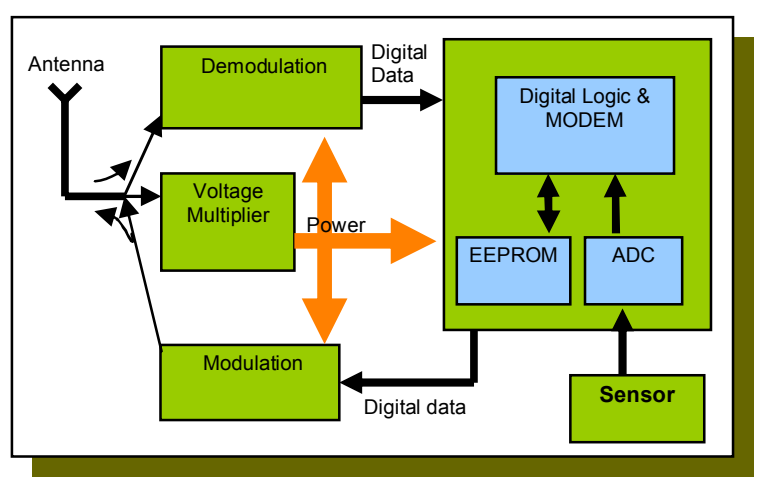

Fig. 3. Block diagram of RFID tag.

Printed RFID tags have been realized in previous work [6]. However; the operating frequency of these printed RFID tags is the Low Frequency (LF) RFID band $(125 \mathrm{KHz}$ and $135 \mathrm{KHz})$; thus they suffer from low read range and a low data rate. The aim is to have an all-printed RFID tag operating at the higher frequencies, such as the UHF $(900 \mathrm{MHz})$ and the microwave RFID frequency bands (above Zigbee's $2.4 \mathrm{GHz}$ and potentially up to $60 \mathrm{GHz}$ ). Results from integrated organic prototypes at $915 \mathrm{MHz}$ (Fig. 4) will be presented at the conference.

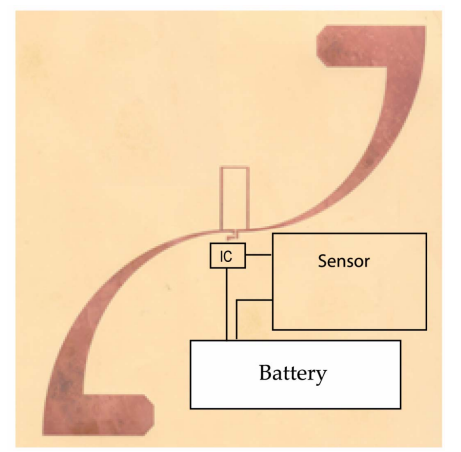

Fig. 4. Suggested outline of integrated S-shaped antenna with IC, sensor and embedded battery.

Paper is considered to be not only the cheapest organic material to humankind but also one of the most environmental friendly ones. Paper can also undergo large reel-to-reel processing. Also another major advantage of this material is that it is compatible with circuit printing by direct write methodologies, such as conductive paste inkjet printing and hence does not require costly etching techniques. This enables modules such as: antennas,
IC, memory, batteries and/or sensors to be easily embedded in. In addition, paper can be made hydrophobic and/or fire-retardant, and it has a dielectric constant $\varepsilon_{\mathrm{r}}(\sim 3)$ close to that of air which allows electromagnetic waves to penetrate easily through the embedded substrate without power losses due to material reflections.

\section{CONCLUSION}

In this paper, we proposed the integration of sensors with UHF RFID's utilizing inkjet printing in/on organic substrates such as paper that will enable the low cost and large-scale implementation of wireless sensor networks. Additionally the use of embedded rechargeable thin film batteries will increase the nodes' lifetime. The use of compact dual-polarization antennas operating in the UHF/RF bands will enable a high read range with high data rate transfer. Altogether, we have introduced a set of technologies which could eventually facilitate a low cost autonomous data acquisition Wireless Sensor Network with inexpensive RFID tags.

\section{REFERENCES}

[1] R. Nohria, R.K. Khillan, Y. Su, R. Dikshit, Y. Lvov, K. Varahramyan "Humidity Sensors based on polyaniline film deposited using layer-by-layer nanoassembly" Sensors and Actuators B, v.114, pp. 218222, 2006

[2] J.N. Palasagaram, R. Ramadoss, "MEMS capacitive pressure sensor array fabricated using printed circuit processing techniques" 32nd Annual Conference of IEEE, Industrial Electronics Society, 2005

[3] M.C. Tanese, D. Fine, A. Dodabalapur, L. Torsi "Organic thin film sensors: Interface dependant and gate bias enhanced responses" Microelectronics journal, v. 37, pp. 837-840, 2006

[4] S. Basat, S. Bhattacharya, A. Rida, S. Johnston, L. Yang, M.M. Tentzeris, and J. Laskar "Fabrication and Assembly of a Novel High-Efficiency UHF RFID Tag on Flexible LCP Substrate" Proc. of the IEEE-ECTC Symposium, San Diego, June 2006

[5] Bates JB, Dudney NJ, Neudecker BJ, Wang B: ThinFilm Lithium Batteries. New Trends in Electrochemical Technology: Energy Storage Systems in Electronics. Singapore: Gordon and Breach; 2000, pp. 453-485.]

[6] Y.J. Chan, C.P. Kung, Z. Pei "Printed RFID: Technology and Application" IEEE International Workshop on Radio Frequency Integration Technology, Singapore, Nov 30- Dec 02, 2005 